\title{
Birth Month and Cardiovascular Disease Risk Association: Is meaningfulness in the eye of the beholder?
}

\author{
Eduard Poltavskiy¹, J. David Spence ${ }^{2}$, Jeehyoung Kim³ ${ }^{3}$, Heejung Bang ${ }^{1,4}$ \\ 1. Graduate Group in Epidemiology, University of California, Davis, CA, USA \\ 2. Stroke Prevention \& Atherosclerosis Research Centre, Robarts Research Institute, \\ Western University, London, Ontario, Canada \\ 3. Department of Orthopedic Surgery, Seoul Sacred Heart General Hospital, Seoul, Korea \\ 4. Division of Biostatistics, Department of Public Health Sciences, University of California, \\ Davis, CA, USA
}

\begin{abstract}
In the modern era, with high-throughput technology and large data size, associational studies are actively being generated. Some have statistical and clinical validity and utility, or at least have biologically plausible relationships, while others may not. Recently, the potential effect of birth month on lifetime disease risks has been studied in a phenome-wide model. We evaluated the associations between birth month and 5 cardiovascular disease-related outcomes in an independent registry of 8,346 patients from Ontario, Canada in 1977-2014. We used descriptive statistics and logistic regression, along with model-fit and discrimination statistics. Hypertension and coronary heart disease (of primary interest) were most prevalent in those who were born in January and April, respectively, as observed in the previous study. Other outcomes showed weak or opposite associations. Ancillary analyses (based on raw blood pressures and subgroup analyses by sex) demonstrated inconsistent patterns and high randomness. Our study was based on a high risk population and could not provide scientific explanations. As scientific values and clinical implications can be different, readers are encouraged to read the original and our papers together for more objective interpretations of the potential impact of birth month on individual and public health as well as toward cumulative/total evidence in general.
\end{abstract}

Key words: birth month; cardiovascular disease; electronic medical record.

Correspondence: hbang@ucdavis.edu

DOI: $10.5210 /$ ojphi.v8i2.6643

Copyright @2016 the author(s)

This is an Open Access article. Authors own copyright of their articles appearing in the Online Journal of Public Health Informatics. Readers may copy articles without permission of the copyright owner(s), as long as the author and OJPHI are acknowledged in the copy and the copy is used for educational, not-for-profit purposes. 


\section{INTRODUCTION}

Possibly beginning with Hippocrates, the environment, including air, water and place has been suggested to influence human health [1]. Numerous researchers hypothesized that pre or peri-natal or early life conditions can have impacts on the occurrence of various diseases over a lifetime. Non-ignorable evidence has been found in some respiratory illness (e.g., asthma) and mental illnesses (e.g., attention deficit hyperactivity disorder, schizophrenia). For example, in the 1970s there was evidence that being born in the winter increased the risk of schizophrenia by 10\% [2-6]. On the other hand, some findings have been claimed and refuted by re-analysis of the same data, and are being used as example in statistical education [7-9]. Nowadays, the volume of related literature is growing and emergence of large, convenient and easily gathered datasets facilitates the analysis of a number of events and potential determinants. So far, the track record has been mixed $[10,11]$.

A recent study assessed whether birth month affects lifetime disease risk of 1,688 conditions in a phenome-wide model based on an electronic medical record (EMR) database, including about 1.75 million individuals, from an institution in New York City from 1900-2000 [12]. The interesting findings generated from the statistical analyses of a huge database received great attention from the scientific community and the media. The authors reported that cardiovascular disease (CVD) was significantly dependent on birth month, asserting that this association was newly discovered in their study. An earlier review of 246 suggested coronary risk factors, including constitutional, demographic and environmental factors, did not include birth month [13]. In contrast, it has been reported that a general tendency for people born in the first half of the year to die at younger age, more from heart disease and cerebrovascular disease, than those born in the second half of the year in Austria [14]. The authors also performed an extensive literature review - 19 out of the 55 identified diseases are supported by the literature - and used rigorous methodologies, including widely accepted statistical adjustment of multiplicity and quality control, which have been common issues in similar studies based on convenient, tertiary datasets that were not collected for research or policy making purposes.

In this paper, we attempt to evaluate the validity and generalizability of their findings in an independent, external patient registry. We focused on 5 CVD-related outcomes: hypertension, coronary heart disease (CHD), stroke, diabetes, and chronic kidney disease (CKD) $[15,16]$. Other outcomes (such as respiratory and reproductive diseases highlighted in the original paper) were not available to us.

\section{MATERIALS and METHODS}

\section{Study population and sample}

The study was conducted using the EMR of the Stroke Prevention \& Atherosclerosis Research Centre, Robarts Research Institute, London, Ontario, with patient visits occurring in 1977-2014. Before 1995, patients were referred to the Hypertension Clinic at Victoria Hospital, London, Canada. Since 1995, they were referred to one of several clinics at University Hospital: a Stroke Prevention Clinic, an Urgent TIA Clinic, and a Premature Atherosclerosis Clinic. Western University Health Science Research Ethics Board approved this study. 


\section{Exposures and outcomes}

We analyzed age and birth month (both in integer), without date or more details. Hypertension was defined as antihypertensive medication usage or systolic blood pressure (BP)>140 $\mathrm{mmHg}$ or diastolic BP>90 mmHg, where the higher value was selected from measurements in the left and right arms. We defined CHD as present if myocardial infarction or vascular surgery was recorded. A cerebrovascular disorder was defined if stroke or transient ischemic attack (TIA) was present; these were combined as Stroke. Diabetes was restricted to type-2 diabetes. CKD was defined using glomerular filtration rate $(\mathrm{GFR})<60$, estimated from the CKD-EPI formula [17].

\section{Data analysis}

We used summary statistics to describe patient characteristics, such as mean, standard deviation and interquartile range for continuous variables, and frequency and percentage for categorical variables. We computed the frequency and (row and column) percentage for each health outcome by birth month. We indicated the highest and lowest percentages, and tested the equality of the proportions over different months by the Chi-square test. Since we utilized EMR data, missing data were common. In all analyses, we included all available data, without imputations, in the included variables in each analysis, and we indicated the sample size.

We fitted Simple logistic regression for each health outcome with each predictor separately. We considered 3 demographics as predictors or independent variables: month, sex, and age, where age was analyzed both as a continuous and dichotomized variable ( $>50 \mathrm{vs}$. $\leq 50$ years) and we did not treat age and sex as confounders in regression. To compare the different models, we employed standard measures for evaluating models and prediction [18-20]: area under the receiver-operatingcharacteristic curve (AUC) and Akaike and Bayesian information criteria (AIC/BIC). AUC is a discrimination statistic; 0.5 means random and 1 means perfect discrimination between cases vs. non-cases. AIC is a measure of the relative quality of a statistical model for given data, and BIC might be considered a Bayesian extension. A lower value of AIC/BIC indicates improved model fit. Some interpret that AIC addresses explanation and BIC addresses prediction [21]. Of note, AIC/BIC do not have a simple range, unlike p-value, correlation or AUC; they should be compared within the same outcome, not across outcomes due to different sample sizes.

As ancillary analyses, we computed the distribution of 4 raw BP measurements (left vs. right, systolic vs. diastolic) over months to examine time-trends, and to check if these measurements support the 'January peak' and 'October trough' for hypertension that were reported in the original study. Also, we fitted the event rate by penalized B-splines by sex in order to see if patterns are similar for men vs. women.

SAS 9.3 was used for analysis (SAS Institute, Cary, NC). P-values and confidence intervals (CIs) are 2-sided and unadjusted for multiplicity.

\section{RESULTS}

Table 1 describes the characteristics of the 8,346 patients included in our study. Patients tended to be older (with mean=63 and range=9-99 years at the first visit to the clinic) and $52 \%$ were male. 
Hypertension was highly prevalent $(66 \%)$, compared to other outcomes $(<25 \%)$. Birth months were quite evenly distributed with the null value of $8.3 \%(=100 / 12)$ overall $(7.5-8.9 \%, \mathrm{p}=0.06)$. Figure 1 presents the event rate of individual health outcomes for each birth month. January (69\%), April (22\%), July (25\%), November (20\%), and March (27\%) showed the highest proportions for hypertension, CHD, stroke, diabetes, and CKD, respectively, and the lowest proportions were in October (63\%), September (13\%), September (20\%), and March (18\%). When we computed the percentage of different birth months among those who had the outcome (i.e., using column percent in place of row percent), the same highest months were observed.

When we modeled different demographic factors as independent variable and different health outcomes as dependent variable by regression, birth month was associated with CHD, diabetes and CKD (mostly for post-hoc selection of the highest month) with $\mathrm{p}=0.02-0.05$. In contrast, sex was highly significant with these 3 outcomes ( $\leq \leq 0.003$ ). Months (without post-hoc dichotomization) yielded slightly higher AUC than sex for hypertension, stroke and CKD, which may imply enhanced discrimination, but AIC/BIC tended to indicate the reversed performance in model fit/quality; see Table 2 .

In the ancillary analysis with raw variables for hypertension, the key findings (January as highest and October as lowest) from the original study were confirmed. On the other hand, we observed that right arm BPs were highest among people who were born in January, whereas left arm BPs were highest in July, which are opposite seasons. Event rate plots by sex revealed less systematic, substantially different trends among men vs. women; see Figure 2.

Table 1. Patient characteristics

\begin{tabular}{|l|l|l|}
\hline Variable & $\begin{array}{l}\text { N of } \\
\text { complete } \\
\text { data }\end{array}$ & $\begin{array}{l}\text { Mean (Standard } \\
\text { deviation) [nterquartile } \\
\text { range] } \\
\text { or Percentage }\end{array}$ \\
\hline Age, years & 8346 & $62.6(14.7)[52.0-74.0]$ \\
\hline Male & 8346 & $51.5 \%$ \\
\hline Height, cm & 6876 & $\begin{array}{l}168.6(10.2)[160.0- \\
176.0]\end{array}$ \\
\hline Weight, kg & 7197 & $78.8(17.7)[66.0-89.1]$ \\
\hline Serum creatinine, mmol/L & 4002 & $87.5(39.0)[69.0-96.0]$ \\
\hline Current smoker & 8217 & $18.3 \%$ \\
\hline Hypertension & 6663 & $66.2 \%$ \\
\hline Diabetes (type 2) & 7971 & $16.4 \%$ \\
\hline Myocardial infarction & 6541 & $11.2 \%$ \\
\hline Vascular surgery & 6566 & $9.6 \%$ \\
\hline
\end{tabular}


Birth Month and Cardiovascular Disease Risk Association: Is meaningfulness in the eye of the beholder?

\begin{tabular}{|l|l|l|}
\hline Stroke & 6855 & $14.4 \%$ \\
\hline Transient ischemic attack & 6751 & $12.3 \%$ \\
\hline Chronic kidney disease* & 4002 & $22.5 \%$ \\
\hline Birth month & 8346 & \\
\hline 1 & & $8.4 \%$ \\
\hline 2 & & $8.1 \%$ \\
\hline 3 & & $8.8 \%$ \\
\hline 4 & & $8.5 \%$ \\
\hline 5 & & $8.6 \%$ \\
\hline 6 & & $8.9 \%$ \\
\hline 7 & & $8.9 \%$ \\
\hline 8 & & $8.2 \%$ \\
\hline 9 & & $8.2 \%$ \\
\hline 10 & & $8.0 \%$ \\
\hline 11 & & $8.2 \%$ \\
\hline 12 & & $7.5 \%$ \\
\hline & & 80794 \\
\hline & & \\
\hline
\end{tabular}

*The CKD-EPI formula was used to estimate glomerular filtration rate; the threshold used to define chronic kidney disease is an eGFR $<60 \mathrm{~mL} / \mathrm{min} / 1.73 \mathrm{~m}^{2}$. 
Birth Month and Cardiovascular Disease Risk Association: Is meaningfulness

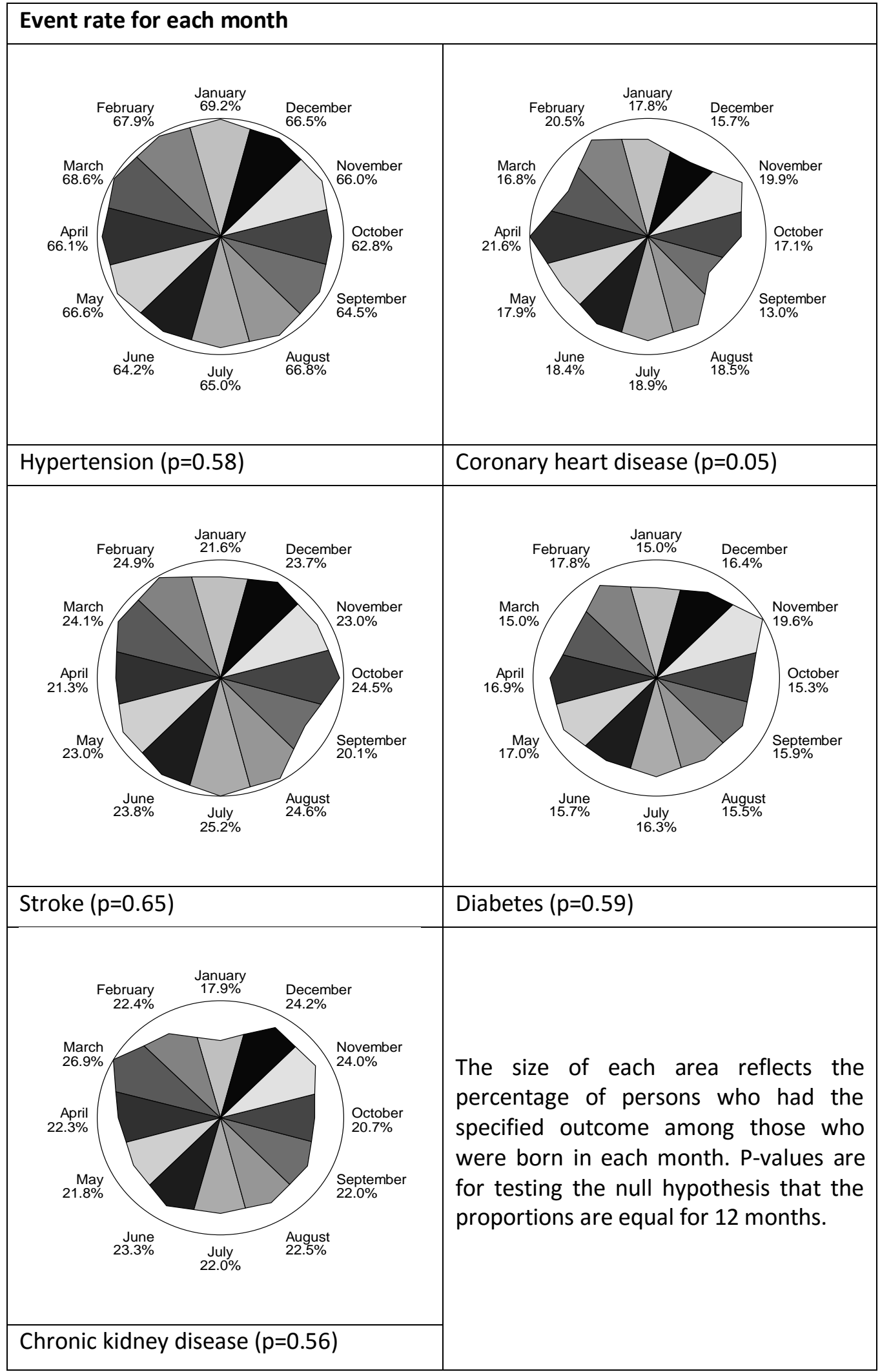

Figure 1. Nightingale plots of the distribution of birth month for health outcomes 
Birth Month and Cardiovascular Disease Risk Association: Is meaningfulness in the eye of the beholder?

a) Time-trends of four blood pressure measurements over month

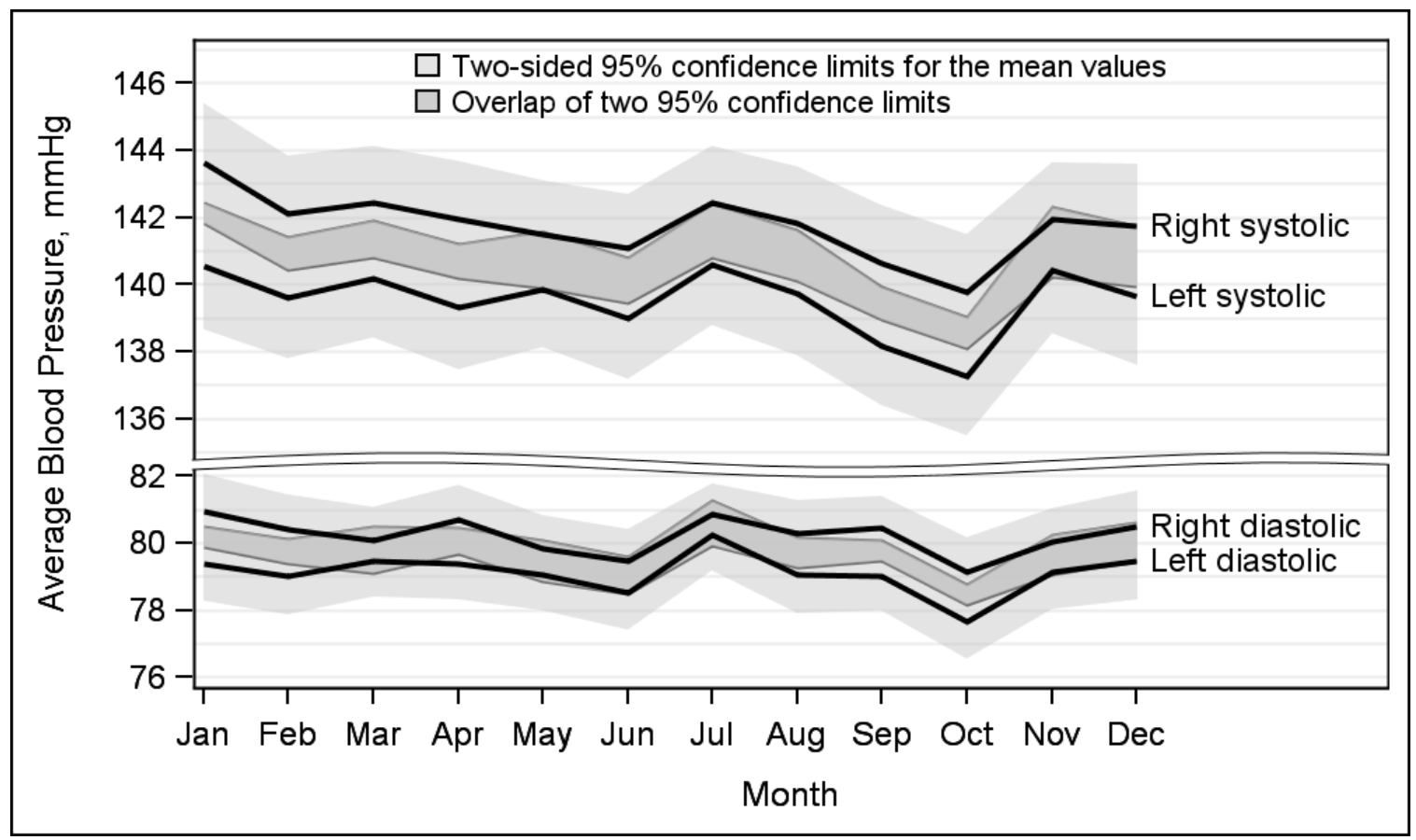

b) Penalized B-spline plot by sex for hypertension and heart disease
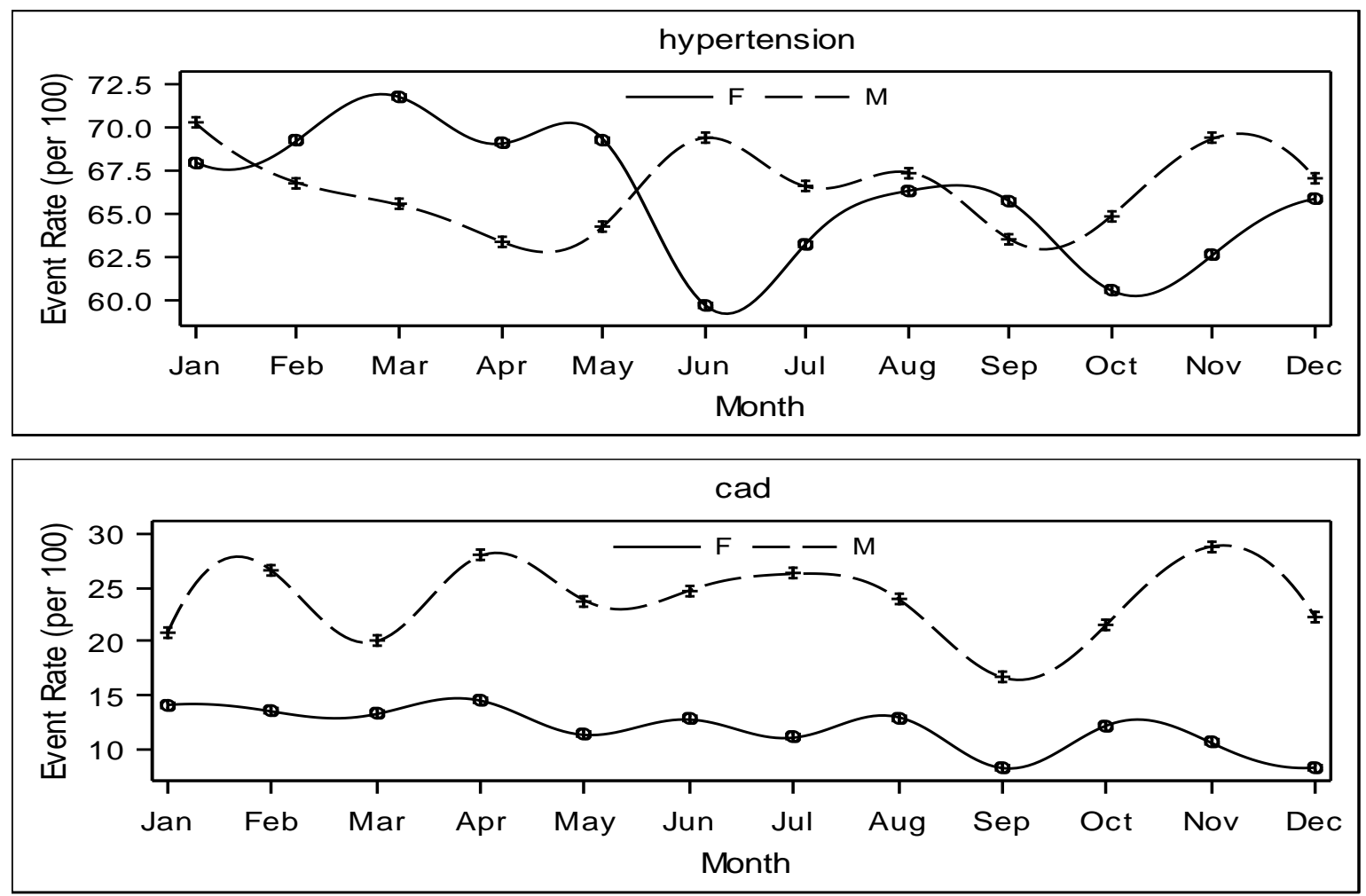

Figure 2. Ancillary analyses 
Birth Month and Cardiovascular Disease Risk Association: Is meaningfulness in the eye of the beholder?

Table 2. Discrimination and model-fit statistics from simple logistic regression

\begin{tabular}{|c|c|c|c|c|c|}
\hline $\begin{array}{l}\text { Health } \\
\text { outcome }\end{array}$ & Predictor & P-value & AUC & AIC & BIC \\
\hline \multirow{5}{*}{$\begin{array}{l}\text { Hypertension } \\
\text { (total } \\
\mathrm{N}=6663 \text { ) }\end{array}$} & Birth months & 0.58 & 0.522 & 8539 & 8621 \\
\hline & Highest month (Jan vs. the rest)* & 0.12 & 0.506 & 8526 & 8540 \\
\hline & Sex & 0.60 & 0.503 & 8528 & 8542 \\
\hline & Age (continuous) & $<0.0001$ & 0.643 & 8124 & 8138 \\
\hline & Age $>50$ & $<0.0001$ & 0.585 & 8285 & 8299 \\
\hline \multirow{5}{*}{$\begin{array}{l}\text { Coronary } \\
\text { heart disease } \\
\text { (total } \\
N=6472 \text { ) }\end{array}$} & Birth months & 0.05 & 0.539 & 6020 & 6102 \\
\hline & $\begin{array}{l}\text { Highest month (April vs. the } \\
\text { rest)* }\end{array}$ & 0.03 & 0.510 & 6016 & 6029 \\
\hline & Sex & $<0.0001$ & 0.598 & 5873 & 5886 \\
\hline & Age (continuous) & $<0.0001$ & 0.621 & 5846 & 5859 \\
\hline & Age $>50$ & $<0.0001$ & 0.572 & 5889 & 5902 \\
\hline \multirow{5}{*}{$\begin{array}{l}\text { Stroke } \\
\text { (total } \\
\mathrm{N}=6845)\end{array}$} & Birth months & 0.66 & 0.523 & 7452 & 7534 \\
\hline & Highest month (July vs. the rest)* & 0.25 & 0.505 & 7440 & 7453 \\
\hline & Sex & 0.94 & $0.509^{+}$ & 7441 & 7455 \\
\hline & Age (continuous) & $<0.0001$ & 0.598 & 7300 & 7313 \\
\hline & Age $>50$ & $<0.0001$ & 0.556 & 7344 & 7358 \\
\hline \multirow{5}{*}{$\begin{array}{l}\text { Diabetes } \\
\text { (total } \\
\mathrm{N}=7971 \text { ) }\end{array}$} & Birth months & 0.59 & 0.525 & 7118 & 7202 \\
\hline & Highest month (Nov vs. the rest)* & 0.02 & 0.510 & 7102 & 7116 \\
\hline & Sex & $<0.0001$ & 0.530 & 7092 & 7106 \\
\hline & Age (continuous) & $<0.0001$ & 0.597 & 6974 & 6988 \\
\hline & Age $>50$ & $<0.0001$ & 0.566 & 6977 & 6991 \\
\hline \multirow{5}{*}{$\begin{array}{l}\text { Chronic } \\
\text { kidney } \\
\text { disease } \\
\text { (total } \\
\mathrm{N}=4002 \text { ) }\end{array}$} & Birth months & 0.57 & 0.530 & 4286 & 4361 \\
\hline & $\begin{array}{l}\text { Highest month (March vs. the } \\
\text { rest)* }\end{array}$ & 0.04 & 0.511 & 4271 & 4284 \\
\hline & Sex & 0.003 & 0.528 & 4267 & 4279 \\
\hline & Age (continuous) & $<0.0001$ & 0.796 & 3457 & 3470 \\
\hline & Age $>50$ & $<0.0001$ & 0.602 & 3991 & 4003 \\
\hline
\end{tabular}

Each predictor is separately modeled as a univariate covariate in Simple logistic regression. 
Birth month (1-12) is included as a categorical covariate (via 11 dummies); sex is binary; and age (in years) is included as a continuous or binary covariate ( $>50$ vs. $\leq 50$ years old).

*Highest month (vs. rest as binary variable) is selected post-hoc, so results may suffer optimism bias.

P-value is computed from Wald Chi-square test; degrees of freedom=11 for birth month and 1 for all others.

AUC, area under the ROC curve, is a discrimination statistic; 0.5 means random discrimination and 1 means perfect discrimination.

AIC, Akaike information criteria, is a measure of the relative quality of a statistical model for a given set of data: a lower value means a better model fit.

BIC, Bayesian information criteria, is a Bayesian extension of AIC: a lower value means a better model fit.

AIC and BIC should be compared within the same outcome due to different Ns and amount of information.

+Estimation issue so we fitted the model with $\mathrm{Y}=$ stroke or TIA, and averaged the AUC of 0.511 and 0.507 .

\section{DISCUSSION}

In the BigData era, with advanced, fancy statistics and informatics tools and highly educated minds, many things that have been impossible are becoming possible. Many small and previously unidentified effects or associations and rare cases are being discovered and reported on a daily basis. At the same time, high standards in data quality and statistical analyses are being emphasized, similarly to Deming's 6-sigma that has been a gold standard in industry and quality control for decades [22,23]. Yet, two different issues are never answered by large sample size, statistical analysis and computing software: 1) clinical or practical meaningfulness (e.g., is the effect size large enough to be clinically meaningful or lead to any action?) and 2) biological plausibility (why does this happen? Is an association of insect bite and birth month with adjusted $\mathrm{p}=0.001$ scientifically explainable?) [12].

Our findings support some of the authors' claims (e.g., hypertension-January and CVD-April with the highest, and September-October with the lowest), which may be regarded as external validation, particularly because London, Ontario and New York City are not very different in climate. But we also found conflicting evidence in related diseases (e.g., January, April, July, November with the highest); so coherence, consistency and plausibility in causal viewpoints might be weakened [24]. Our analysis demonstrates high randomness going on, which may be natural. For example, the phenomena of 'right arm BP highest in January and left arm BP highest in July' and of the differential effects of birth month for males vs. females are not biologically plausible. Can subdiseases/conditions within the same disease category be qualitatively different and be associated with different months? Small but real differences or being 'fooled by randomness' cannot be excluded [25]. 
The observed AUCs, a key measure in prediction, are tantalizing, accepting that the role of age is fully known. For hypertension and stroke, month may offer better discrimination than sex, but model-fit seems to show that sex could be better. Birth month did not increase discrimination ability for all outcomes once age and sex are included in the model; AUC increase $=0.001$ for hypertension and 0 for others (Results not shown). Large data sets cause impressive p-values with minor differences in biology. Are they clinically relevant [13,22,26,27]? Despite substantially different study populations and sample sizes, dramatically different $\mathrm{p}$-values for two validated outcomes (CHD and hypertension) are noteworthy: p-values $<0.001$ adjusted for 1,688 comparisons (or p-value $\sim 10^{-22}$ unadjusted using our best guess from the Manhattan plot) in the original study vs. unadjusted p-values $=0.03-0.58$ in our study [28,29]. When a number of p-values - probably the most popular statistical measure in research - are computed, a simple ' $p$-value plot' together with AUC could be helpful for assessing overall randomness in associations [8,30,31]. Related to the recent 'bad luck-cancer controversy', the validity and proper interpretation of another popular statistic, $\mathrm{R}^{2}$, for aggregated data have been discussed [32].

The limitations of our study and caveats for readers should be noted. First, we utilized retrospective data from a high-risk sample in a single geographical region, which could make already small associations even smaller. Very large population-based cohort or census would be ideal. Second, we are unable to explain some findings and to identify causes or underlying mechanisms; yet these issues are shared by the original, our and many other non-experimental studies. Third, the common goal of a long history of birth month research could be different from ours; its goal is generally a basic or pure science one to find diseases that may be related to developmental effects of environmental exposures, which presumably would later be investigated to elucidate the mechanism of that association. In contrast, our goal is closer to a clinical practice one, which may be better addressed by a statistical or prediction measure such as AUC, in addition to or place of p-value. For example, should physicians or patients be more suspicious of and investigate more closely for certain conditions based on birth month? (e.g., screening); should parent planning a pregnancy aim to have their child born in a certain month? Indeed, significant seasonality but different seasons/months for the best outcomes with high randomness have been demonstrated in infertility, autism and mortality-related research as well [2,14,33-37].

The main strengths are: a relatively large sample size covering a long term from multiple hospitals; multiple CVD-related outcomes; use of clinical data (e.g., multiple raw BPs in place of coded data where underreporting can be severe) and EMR with continuous quality checks [38-42]; and statistical measures that address different aspects of model and association, beyond p-value. Since our cohort mostly consists of older adults, the 'lifetime risk' of CVD that the original study intended to address might be well-captured although representativeness is weaker.

Scientists and readers' efforts to confirm important findings and attitudes to wait for more evidence should be valued more, in addition to discovery, innovation and productivity that are currently emphasized [22]. It is well documented that the Framingham risk score - a landmark in CVD research - does not perform well in Asian populations or HIV patients [43]. We do not think this is a major weakness of the method/finding as no method is perfect and virtually no finding is universal. Also, for every finding, we need to determine whether it is real vs. not (e.g., random), 
and if real, the next step might be to assess biological mechanisms as well as practical value and clinical implications.

\section{CONCLUSIONS}

We could validate the associations between birth month and the two primary CVD-related outcomes, but also found randomness was high. Until a definitive or ultimate answer, which may be provided from very large, representative samples with accurate outcomes data covering different climates, physicians and patients need not be much concerned about birth month; modifiable factors are a more appropriate focus. When faced with reports of novel discoveries, healthy skepticism and waiting for validations and explanations in similar and different settings are crucial for citizens in the Information Age. Finally, we still believe that EMR offers invaluable resources and opened a new chapter in research and data science. The following quotation, often attributed to Galileo Galilei, is apt: Measure what is measurable; make measurable what is not so [44]. Perhaps clinical and lab data are more suited to the first task, while administrative or selfreport data try to do the latter (as the next best option).

\section{Acknowledgements}

We thank Drs. Robert Elston, Dmitri Zaykin, Stan Young, and Allan Jaffe for providing useful comments.

\section{Conflict of interests:}

None declared.

\section{Funding:}

H. Bang was partly supported by the National Center for Advancing Translational Sciences, National Institutes of Health, through grant number UL1 TR000002.

\section{References}

1. Hippocrates. On Airs, Waters, and Places. Accessed on 09/2016; Available from: http://classics.mit.edu/Hippocrates/airwatpl.mb.txt.

2. Zerbo O, et al. 2011. Month of conception and risk of autism. Epidemiology. 22(4), 469-75. http://dx.doi.org/10.1097/EDE.0b013e31821d0b53

3. Kauhanen L, et al. 2006. Social disadvantages in childhood and risk of all-cause death and cardiovascular disease in later life: a comparison of historical and retrospective childhood information. Int J Epidemiol. 35(4), 962-68. http://dx.doi.org/10.1093/ije/dyl046

4. Torrey EF, et al. 1997. Seasonality of births in schizophrenia and bipolar disorder: a review of the literature. Schizophr Res. 28(1), 1-38. http://dx.doi.org/10.1016/S0920-9964(97)00092$\underline{3}$ 
5. Gallagher BJ, III, et al. 2007. Schizophrenic subtype, seasonality of birth and social class: a $\begin{array}{lllll}\text { preliminary } & \text { analysis. } & \text { Eur } & \text { Psychiatry. } & 22(2),\end{array}$ http://dx.doi.org/10.1016/j.eurpsy.2006.07.004

6. Templcr DI. 1982. Comment on the Lewis and Griffin explanation for season of birth effect in schizophrenia. Orthomolecular Psychiatry. 11(3), 219-21.

7. Mathews F, Johnson PJ and Neil A. 2008. You are what your mother eats: evidence for maternal preconception diet influencing foetal sex in humans. Proc Biol Sci. 275(1643), 1661-8.

8. Young SS, Bang H, and K. Oktay K. 2009. Cereal-induced gender selection? Most likely a multiple testing false positive. Proc Biol Sci. 276(1660): 1211-2; discussion 1213-4.

9. Utts J. Unintentional lies in the media: don't blame journalists for what we don't teach, in Proceedings of the Eighth International Conference on Teaching Statistics, In C. Reading (Ed.), Data and Context in Statistics Education. 2010: Voorsburg, The Netherlands: International Statistical Institute.

10. Salzberg S. Why Google Flu is a Failure. 2014. Accessed on 09/2016; Available from: http://www.forbes.com/sites/stevensalzberg/2014/03/23/why-google-flu-is-a-failure/.

11. Sliver N. The Signal and the Noise: Why So Many Predictions Fail - but Some Don't. 2015: Penguin Books.

12. Boland MR, et al. 2015. Birth month affects lifetime disease risk: a phenome-wide method. JAMIA. 22(5), 1042-1053.

13. Hopkins PN, Williams RR. 1981. A survey of 246 suggested coronary risk factors. Atherosclerosis. 40(1), 1-52. http://dx.doi.org/10.1016/0021-9150(81)90122-2

14. Doblhammer G, Vaupel J. 2001. Lifespan depends on month of birth. Proc Natl Acad Sci USA. 98(5), 2934-39. http://dx.doi.org/10.1073/pnas.041431898

15. Grundy SM, et al. 1999. Diabetes and cardiovascular disease: a statement for healthcare professionals from the American Heart Association. Circulation. 100(10), 1134-46. http://dx.doi.org/10.1161/01.CIR.100.10.1134

16. Weiner DE, Sarnak MJ. 2007. Chronic kidney disease and cardiovascular disease: A bidirectional relationship? Dial Transplant. 36(3), 113-20. http://dx.doi.org/10.1002/dat.20104

17. Levey AS, et al. 2009. CKD-EPI (Chronic Kidney Disease Epidemiology Collaboration). A new equation to estimate glomerular filtration rate. Ann Intern Med. 150(9), 604-12. http://dx.doi.org/10.7326/0003-4819-150-9-200905050-00006

18. Bang H, et al. 2009. Screening for kidney disease in vascular patients: SCreening for Occult REnal Disease (SCORED) experience. Nephrol Dial Transplant. 24(8), 2452-57. http://dx.doi.org/10.1093/ndt/gfp124 
19. Cook NR, Buring JE, Ridker PM. 2006. The effect of including C-reactive protein in cardiovascular risk prediction models for women. Ann Intern Med. 145(1), 21-29. http://dx.doi.org/10.7326/0003-4819-145-1-200607040-00128

20. Steyerberg E. Clinical Prediction Models. 2009: Springer.

21. Shmueli G. 2010. To explain or to predict? Stat Sci. 25, 289-310. http://dx.doi.org/10.1214/10$\underline{\text { STS330 }}$

22. Horton R. 2015. Offline: What is medicine's 5 sigma? Lancet. 385(9976), 1380. http://dx.doi.org/10.1016/S0140-6736(15)60696-1

23. Liao L, Mark DH. 2003. Clinical prediction models: Are we building better mousetraps? J Am Coll Cardiol. 42(5), 851-53. http://dx.doi.org/10.1016/S0735-1097(03)00836-2

24. Hill AB. 1965. The environment and disease: association or causation? Proc R Soc Med. 58, 295-300.

25. Taleb NN. Fooled by Randomness: the Hidden Role of Chance in Life and in the Markets. 2005, Random House Trade Paperbacks.

26. Horton R. 2000. From star signs to trial guidelines. Lancet. 355(9209), 1033-34. http://dx.doi.org/10.1016/S0140-6736(00)02031-6

27. Austin PC, et al. 2006. Testing multiple statistical hypotheses resulted in spuriou s associations: a study of astrological signs and health. $J$ Clin Epidemiol. 59(9), 964-69. http://dx.doi.org/10.1016/j.jclinepi.2006.01.012

28. Boos D, Stefanski L. 2011. P-value precision and reproducibility. Am Stat. 65(4), 231-221. http://dx.doi.org/10.1198/tas.2011.10129

29. Kim J, Bang H. 2016. Three common misuses of p-values. Dent Hypotheses. 7(3), 73-80.

30. Schweder T, Spjøtvoll E. 1982. Plots of P-values to evaluate many tests simultaneously. Biometrika. 69(3), 493-502. http://dx.doi.org/10.1093/biomet/69.3.493

31. Wasserstein RL, Lazar NA. 2016. The ASA's statement on p-values: context, process, and purpose. Am Stat. 70(2), 129-133. http://dx.doi.org/10.1080/00031305.2016.1154108

32. Weinberg C, Zaykin D. 2015. Is bad luck the main cause of cancer? J Natl Cancer Inst. 107(7), djv125. http://dx.doi.org/10.1093/jnci/djv125

33. Rojansky N, et al. 2000. Seasonal variability in fertilization and embryo quality rates in women undergoing IVF. Fertil Steril. 74(3), 476-81. http://dx.doi.org/10.1016/S0015$\underline{0282(00) 00669-5}$ 
34. Stolwijk AM, et al. 1994. Infertility: Seasonality in the results of in-vitro fertilization. Hum Reprod. 9(12), 2300-5.

35. Wood S, et al. 2006. Seasonal variation in assisted conception cycles and the influence of photoperiodism on outcome in in vitro fertilization cycles. Hum Reprod. 9(4), 223-29.

36. Barak Y, et al. 1995. Season of birth and autistic disorder in Israel. Am J Psychiatry. 152(5), 798-800. http://dx.doi.org/10.1176/ajp.152.5.798

37. Kannisto V, Christensen K, Vaupel J. 1997. No increased mortality in later life for cohorts born during famine. Am $J$ Epidemiol. 145(11), 987-94. http://dx.doi.org/10.1093/oxfordjournals.aje.a009067

38. Bogiatzi C, Spence JD. 2012. Ezetimibe and regression of carotid atherosclerosis: importance of measuring plaque burden. Stroke. 43(4), 1153-55. http://dx.doi.org/10.1161/STROKEAHA.111.640789

39. Iemolo F, et al. 2004. Sex differences in carotid plaque and stenosis. Stroke. 35(2), 477-81. http://dx.doi.org/10.1161/01.STR.0000110981.96204.64

40. Spence JD. 2015. Blood pressure control in Canada: the view from a stroke prevention clinic. Can J Cardiol. 31(5), 593-95. http://dx.doi.org/10.1016/j.cjca.2015.03.005

41. Woo J, et al. 2009. Obesity identified by discharge ICD-9 codes underestimates the true prevalence of obesity in hospitalized children. $J$ Pediatr. 154(3), 327-31. http://dx.doi.org/10.1016/j.jpeds.2008.09.022

42. Perkins DO, Wyatt RJ, Barkto JJ. 2000. Penny-wise and pound-foolish: the impact of measurement error on sample size requirements in clinical trials. Biol Psychiatry. 47, 762-66. http://dx.doi.org/10.1016/S0006-3223(00)00837-4

43. Myerson M, et al. 2014. Prevalence, treatment, and control of dyslipidemia and hypertension in 4278 HIV outpatients. $J$ Acquir Immune Defic Syndr. 66, 370-77. http://dx.doi.org/10.1097/QAI.0000000000000168

44. Kleinert A. 2009. Dermessende Luchs. Zwei verbreitete Fehler in der Galilei-Literatur. NTM Zeitschrift für Geschichte der Wissenschaften. Technik und Medizin. 17(2), 199-206. doi:10.1007/s00048-009-0335-4 